\title{
Vascular Contributions to Neurodegeneration: Protocol of the COMPASS-ND Study
}

\author{
Eric E. Smith (D), Simon Duchesne, Fuqiang Gao, Feryal Saad, Victor Whitehead, \\ Cheryl R. McCreary, Richard Frayne, Serge Gauthier, Richard Camicioli, \\ Michael Borrie, Sandra E. Black
}

\begin{abstract}
Objective: To describe the neuroimaging and other methods for assessing vascular contributions to neurodegeneration in the Comprehensive Assessment of Neurodegeneration and Dementia (COMPASS-ND) study, a Canadian multi-center, prospective longitudinal cohort study, including reliability and feasibility in the first 200 participants. Methods: COMPASS-ND includes persons with Alzheimer's disease (AD; $n=150$ ), Parkinson's disease (PD) and Lewy body dementias (LBDs) (200), mixed dementia (200), mild cognitive impairment (MCI; 400), subcortical ischemic vascular MCI (V-MCI; 200), subjective cognitive impairment (SCI; 300), and cognitively intact elderly controls (660). Magnetic resonance imaging (MRI) was acquired according to the validated Canadian Dementia Imaging Protocol and visually reviewed by either of two experienced readers blinded to clinical characteristics. Other relevant assessments include history of vascular disease and risk factors, blood pressure, height and weight, cholesterol, glucose, and hemoglobin A1c. Results: Analyzable data were obtained in 197/200 of whom 18 of whom were clinically diagnosed with V-MCI or mixed dementia. The overall prevalence of infarcts was $24.9 \%$, microbleeds was $24.6 \%$, and high white matter hyperintensity (WMH) was $31.0 \%$. MRI evidence of a potential vascular contribution to neurodegeneration was seen in $12.9 \%-40.0 \%$ of participants clinically diagnosed with another condition such as AD. Inter-rater reliability was good to excellent. Conclusion: COMPASS-ND will be a useful platform to study vascular brain injury and its association with risk factors, biomarkers, and cognitive and functional decline across multiple age-related neurodegenerative diseases. Initial findings show that MRI-defined vascular brain injury is common in all cognitive syndromes and is under-recognized clinically.
\end{abstract}

RÉSUMÉ : Impact des lésions cérébrales vasculaires dans des cas de neuro-dégénération : le protocole de l'étude COMPASS-ND. Objectif : Décrire le rôle de la neuro-imagerie et celui d'autres méthodes dans l'évaluation de l'impact des lésions cérébrales vasculaires (LCV) dans des cas de neuro-dégénération, et ce, dans le cadre de l'étude Comprehensive Assessment of Neurodegeneration and Dementia (COMPASS-ND). Menée dans plusieurs établissements de santé du Canada, il s'agit d'une étude de cohorte longitudinale prospective ayant inclus des critères de fiabilité et de faisabilité appliqués aux 200 premiers participants. Méthodes : L'étude COMPASS-ND a inclus des individus atteints de la maladie d'Alzheimer (MA ; $n=150$ ), de la maladie de Parkinson (MP), de démence à corps de Lewy (DCL ; $n=200$ ), de démence mixte (DM ; $n=200)$, de troubles cognitifs légers (TCL; $n=400$ ), de TCL attribuables à un accident ischémique vasculaire de la région sous-corticale (TCL-V ; $n=200$ ), de troubles cognitifs subjectifs (TCS ; $n$ $=300)$ ainsi qu'un groupe de témoins âgés en santé sur le plan cognitif $(n=660)$. À noter que nos IRM ont été acquises selon le Protocole canadien d'imagerie de la démence (PCID) et ont été ensuite passées en revue visuellement en double aveugle par l'un ou l'autre de nos évaluateurs expérimentés en ce qui concerne les caractéristiques cliniques en jeu. D'autres évaluations pertinentes ont inclus l'historique de maladie vasculaire des individus à l'étude de même que leurs facteurs de risque, leur pression artérielle, leur taille et leur poids, leur taux de cholestérol, de glucose et d'hémoglobine A1c. Résultats : Des données analysables ont pu être obtenues chez 197 individus sur 200. Sur ces 197 individus, on en a diagnostiqué 18 avec des TCL-V ou une forme de démence mixte. La prévalence générale des infarctus s'est établie à $24,9 \%$; celle des microhémorragies du cerveau à $24,6 \%$; et celle des hyperintensités de la matière blanche à $31,0 \%$. Les preuves par IRM d'un potentiel impact des LCV en matière de neuro-dégénération ont été observées chez 12,9 à 40,0 \% des individus chez qui l'on avait diagnostiqué cliniquement une autre condition médicale que la MA. Enfin, soulignons que la fiabilité interévaluateurs s'est avérée bonne à excellente. Conclusion : Le protocole de l'étude COMPASS-ND s'est révélé une plate-forme utile pour se pencher sur les $\mathrm{LCV}$ et sur leurs liens avec certains facteurs de risque, biomarqueurs et tendances à un déclin cognitif et fonctionnel dans le cas de multiples maladies

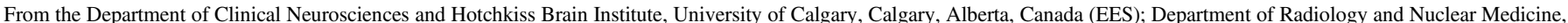

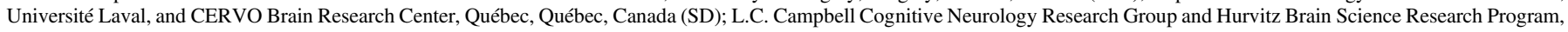

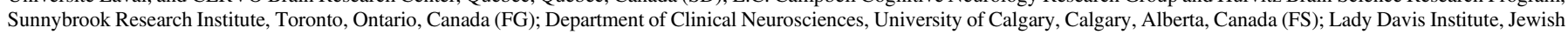

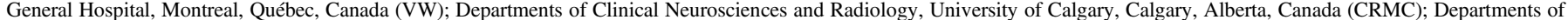

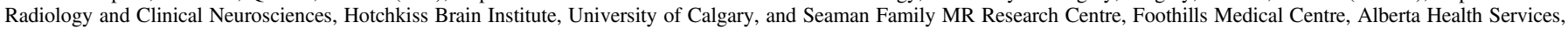

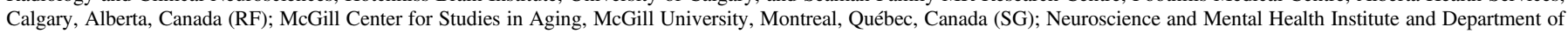

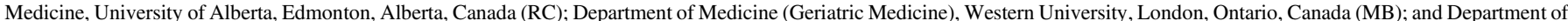

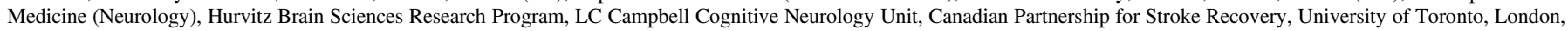
Ontario, Canada (SEB)

Received October 16, 2020. Final Revisions Submitted January 3, 2021. Date of Acceptance January 18, 2021.

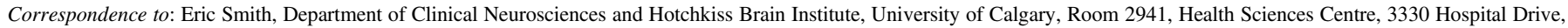

Calgary, Alberta T2N 4N1, Canada. Email: eesmith@ucalgary.ca 
neurodégénératives liées au vieillissement. Des premiers résultats montrent à cet égard que les LCV décrites par IRM sont communes à tous les syndromes cognitifs et ne sont pas suffisamment reconnues sur le plan clinique.

Keywords: Dementia, cerebral small vessel disease, brain infarction, leukoaraiosis, vascular dementia doi:10.1017/cjn.2021.19

\section{INTRODUCTION}

Neurodegeneration has many causes. However, there is increasing evidence that different causes of neurodegeneration share similar molecular mechanisms and converge on shared pathways to cause network dysfunction and cognitive decline. The Comprehensive Assessment of Neurodegeneration and Dementia (COMPASS-ND) study is a Canada-wide prospective cohort study to investigate multiple different causes of neurodegeneration, and their interactions, in a single study. with a comprehensive protocol. ${ }^{1}$

One of the most common contributors to neurodegeneration and cognitive decline is cerebrovascular disease, which can lead to vascular brain lesions that cause cognitive impairment. In this paper, we describe the methods for investigating vascular brain lesions in the COMPASS-ND study, including magnetic resonance imaging (MRI) measurement. Although the overall protocol for COMPASS-ND has been published, it did not include the methods for MRI review and their reliability. ${ }^{1}$ Initial findings from the first wave of study 200 participants are presented. We hypothesized that vascular brain injury would be common across all cognitive disorders even if not recognized at the time of enrollment.

\section{Methods}

\section{Patient Population}

COMPASS-ND is a Canada-wide multi-center prospective, longitudinal cohort study. The overall study design and methods have been published previously. ${ }^{1}$ The study is registered on clinicaltrials.gov (NCT03402919). In brief, the objective is to create a platform for research on neurodegenerative and vascular causes of mild cognitive impairment (MCI) and dementia. Recruitment began in 2016 and is expected to complete in approximately 2023. The study is conducted with ethical approval by institutional review boards at each site, with written informed consent of the participants. In this manuscript we report the methods for assessing vascular contributions to neurodegeneration, with interim findings.

In total, 2310 participants will be recruited in these groups: cognitively intact elderly $(n=660)$, subjective cognitive impairment (SCI; 300), MCI (400), subcortical ischemic vascular MCI (V-MCI; 200), mild dementia due to clinically probable Alzheimer's disease (AD; 150), mild dementia of mixed etiology (200), Parkinson's disease (PD) and Lewy body dementias (LBDs; 200), and frontotemporal dementia (200). Full selection criteria have been published previously. ${ }^{1}$ Participants are being recruited, generally from specialty cognitive clinics, at 31 sites across Canada.

Diagnoses are made by the site investigator based on all available clinical and research information available at the time of assessment. A screening diagnosis is made based on the information from the referral and screening along with referral information including clinical neuroimaging, and then a clinical diagnosis is made additionally incorporating information collected at the research clinical and physical assessment but prior to research MRI or other biomarker information. Group analyses in this paper are based on the clinical diagnosis groupings.

Based on a priori scientific interest, two groups of patients with vascular cognitive impairment were identified using specific selection criteria: subcortical ischemic V-MCI, and mixed etiology dementia with a vascular component. Criteria for V-MCI were derived from consensus criteria from the American Heart Association $^{2}$ and International Society for Vascular Cognitive and Behavioral Disorders (Vas-Cog). ${ }^{3}$ V-MCI participants were required to be age $\geq 60$, have $\mathrm{MCI}$ according to National Institute on Aging-Alzheimer's Association criteria (NIA-AA), ${ }^{4}$ not have a prior history of clinical stroke, and to have evidence of cerebrovascular disease on brain computed tomography (CT) or MRI defined as two or more supratentorial infarcts (i.e., excluding brainstem or cerebellar infarcts) or beginning confluent or confluent white matter hyperintensity (WMH) according to the Age-Related White Matter Change (ARWMC) scale $^{5}$ on any slice. For the V-MCI group, participants with history of symptomatic stroke were excluded so that the focus is on V-MCI caused by covert cerebrovascular disease, predominantly due to cerebral small vessel disease. Criteria for mixed dementia were adapted from NIA-AA criteria for dementia due to $\mathrm{AD}^{6}$ and required that a non-AD cause of dementia should additionally be present. Although the presence of vascular disease was not required for the mixed dementia group, in practice most cases of mixed dementia have a vascular component. ${ }^{7}$ Persons with symptomatic stroke within the last year were excluded. Full details of the study inclusion and exclusion criteria are provided in the online-only supplement (online Table I).

\section{Measurements and Outcomes}

Data on age, sex, past medical and surgical history, medications, tobacco and alcohol intake, hearing, and vision are collected. Cognitive and neurobehavioral measurements include the Montreal Cognitive Assessment, ${ }^{8}$ Clinical Dementia Rating Scale, ${ }^{9}$ Mild Behavioral Impairment Checklist ${ }^{10}$ and Apathy questionnaire. The Hachinski Ischemic Score ${ }^{11}$ is collected. A two-hour neuropsychological test battery is administered. Full details of these assessments have been published previously. ${ }^{1}$ Participants will be followed for two years with a repeat of these assessments at two years. At this time, repeat brain imaging is not planned.

Physiological measurements include sitting and orthostatic blood pressure, height and weight (to calculate body mass index), and waist and hip circumference. Blood measurements include complete blood count, creatinine, hemoglobin A1c, insulin, homocysteine, C-reactive protein, high-density and low-density lipoprotein (HDL and LDL), and apolipoprotein B. Lumbar puncture is offered as a voluntary option and will be analyzed 


\section{Table 1: COMPASS-ND assessments of interest for vascular cognitive impairment}

\begin{tabular}{|c|c|c|}
\hline Domain & Variables & Comments \\
\hline Medical history & History of stroke, hypertension, diabetes & Includes duration of illness \\
\hline Lifestyle habits & Smoking and alcohol use & \\
\hline Neurological exam & Focal signs, tone, frontal signs & \\
\hline VCI diagnostic score & Hachinksi Ischemic Score & \\
\hline Gait and balance & $\begin{array}{l}\text { Number of falls in last year, Activities Specific Balance Confidence (ABC) Scale, timed } 6 \mathrm{~m} \text { walk } \\
\text { with dual task }\end{array}$ & \\
\hline Diet & Food frequency questionnaire & \\
\hline Physical activity & $\begin{array}{l}\text { Physical Activity Scale for the Elderly, California Teachers Study Long Term Recreational } \\
\text { Physical Activity Survey }\end{array}$ & \\
\hline Function & $\begin{array}{l}\text { Clinical Dementia Rating scale, Lawton-Brody scale, Modified Older Americans Resources and } \\
\text { Services (OARS) Activities of Daily Living }\end{array}$ & \\
\hline Behavior & $\begin{array}{l}\text { Mild Behavioral Impairment Checklist, Neuropsychiatric Inventory Questionnaire, Apathy } \\
\text { Questionnaire }\end{array}$ & \\
\hline Cognition & Montreal Cognitive Assessment, neuropsychological test battery & \\
\hline Microbiome & & Fecal and samples stored for later use \\
\hline Physiological measurements & Height, weight, waist and hip circumference, grip strength, orthostatic blood pressure & \\
\hline AD markers & CSF amyloid beta $1-42$, total tau, phosphorylated tau & Optional, aiming for $\geq 25 \%$ of participants \\
\hline Blood markers & Glucose, hemoglobin A1c, total cholesterol, LDL, HDL, apolipoprotein-A1, apolipoprotein-B & \\
\hline
\end{tabular}

Full details of the neuropsychological test battery have been published previously. ${ }^{1}$

Table 2: Canadian Dementia Imaging Protocol ${ }^{12}$

\begin{tabular}{l|c|l|l}
\hline Sequence & Voxel size $(\mathbf{x} / \mathbf{y} / \mathbf{z})\left(\mathbf{m m}^{3}\right)$ & Comments & Role in vascular assessment \\
\hline 3D-T1 & $1.0 \times 1.0 \times 1.0$ & & Volume, cortical thickness, infarcts \\
\hline T2-weighted & $0.94 \times 0.94 \times 3.0$ & & Infarcts, WMH, perivascular spaces \\
\hline Proton density & $0.94 \times 0.94 \times 3.0$ & & Infarcts, WMH \\
\hline T2 FLAIR & $0.94 \times 0.94 \times 3.0$ & & WMH, infarcts \\
\hline T2*-weighted GRE & $0.94 \times 0.94 \times 3.0$ & & Microbleeds cSS \\
\hline Diffusion tensor imaging & $2.0 \times 2.0 \times 2.0$ & 30 directions, $\mathrm{b}=1000 \mathrm{~s} / \mathrm{mm}^{2}$ & $\begin{array}{c}\text { White matter connectivity and mean } \\
\text { diffusivity }\end{array}$ \\
\hline BOLD & $3.5 \times 3.5 \times 3.5$ & 300 volumes, 11 minutes & Functional connectivity
\end{tabular}

FLAIR = fluid attenuated inversion recovery; GRE $=$ gradient-recalled echo; BOLD = blood oxygen level dependent.

for beta-amyloid, total tau, and phosphorylated tau. Additional measurements potentially relevant to defining vascular contributions to neurodegeneration are shown in Table 1.

Brain MRI are acquired using the previously validated Canadian Dementia Imaging Protocol (www.cdip-pcid.ca). ${ }^{12,13}$ The protocol is summarized in Table 2. Compliant with the Standards for Reporting Vascular Changes on Imaging (STRIVE), ${ }^{14}$ it was harmonized on three major vendor platforms and includes a common procedure for qualification, quality control, and quality assurance.

A Vascular Core Lab for visual review of brain MRI is distributed between the University of Calgary and Sunnybrook Hospital (University of Toronto). Each MRI is visually reviewed for evidence of vascular brain injury by one of two trained readers qualified by the Vascular Core Lab. Readers are required to be neurologists or radiologists with more than five years of clinical experience with cerebrovascular lesions on MRI, to complete a training module, and to exhibit good inter-rater reliability on a training set (kappa $\geq 0.60$ ) for common vascular lesions (presence of brain infarcts, presence of microbleeds, and Fazekas WMH rating scale ${ }^{15}$ ). MRI scans are reviewed, blinded to clinical information, using a standardized case report form. Infarcts, microbleeds, and WMH are defined according to STRIVE. ${ }^{14}$ Infarcts are classified by size, number, location, and vascular territory, which allows their subdivision as lacunar versus non-lacunar type. Microbleeds are classified by number and location as in the Brain Observer Microbleeds (BOMBS) scale. ${ }^{16}$ If microbleeds or cortical superficial siderosis are present their pattern is classified as probably, possibly, or not consistent with cerebral amyloid angiopathy as defined by revised Boston criteria. ${ }^{17}$ When lobar microbleeds or cortical superficial siderosis were accompanied by non-lobar 
Table 3: Characteristics of the First 200 participants

\begin{tabular}{|c|c|c|c|c|c|c|c|c|}
\hline Characteristic & All $(n=197)$ & $\begin{array}{c}\text { Alzheimer's } \\
\text { disease } \\
\text { dementia } \\
(n=24)\end{array}$ & $\begin{array}{c}\text { Mixed/V-MCI } \\
\quad(n=18)\end{array}$ & $\operatorname{MCI}(n=84)$ & SCI $(\mathbf{n}=35)$ & $\begin{array}{c}\text { PD/LBD } \\
(\mathbf{n}=\mathbf{3 1})\end{array}$ & Other $(\mathbf{n}=5)$ & $P$ value \\
\hline Age & $73.4(7.0)$ & $76.6(7.2)$ & $79.4(3.8)$ & $73.4(6.5)$ & $69.4(6.2)$ & $72.2(7.2)$ & $69.9(7.6)$ & $<0.001$ \\
\hline Female sex & 44.1 & 12.5 & 33.3 & 51.2 & 74.3 & 22.6 & 40.0 & $<0.001$ \\
\hline University education & 44.7 & 45.8 & 55.6 & 40.5 & 48.6 & 45.2 & 40.0 & 0.88 \\
\hline Body mass index & $26.3(4.4)$ & $27.1(4.2)$ & $25.7(3.5)$ & $26.5(4.8)$ & $26.7(5.1)$ & $25.5(3.0)$ & $24.9(1.8)$ & 0.73 \\
\hline Waist:hip ratio & $0.95(0.13)$ & $1.0(0.2)$ & $0.97(0.1)$ & $0.95(0.1)$ & $0.91(0.1)$ & $0.95(0.1)$ & $1.05(0.1)$ & 0.04 \\
\hline Systolic BP & $131(17)$ & $121(13)$ & $138(16)$ & $134(18)$ & $127(15)$ & $129(15)$ & $150(15)$ & $<0.001$ \\
\hline Diastolic BP & $73(9)$ & $68(7)$ & $74(10)$ & $74(8)$ & $71(10)$ & $77(9)$ & $79(7)$ & 0.004 \\
\hline Stroke & 4.1 & 4.2 & 11.1 & 3.6 & 0 & 6.5 & 0 & 0.49 \\
\hline Hypertension & 39.1 & 33.3 & 66.7 & 36.9 & 22.9 & 51.6 & 40.0 & 0.03 \\
\hline Diabetes & 10.3 & 8.7 & 16.7 & 13.3 & 2.9 & 10.0 & 0 & 0.51 \\
\hline Hypercholesterolemia & 46.2 & 33.3 & 66.7 & 54.8 & 25.7 & 38.7 & 80.0 & 0.007 \\
\hline Coronary heart disease & 15.3 & 12.5 & 44.4 & 14.3 & 5.9 & 16.1 & 0 & 0.009 \\
\hline $\mathrm{MoCA}$ & $23.3(4.2)$ & $19.3(3.0)$ & $21.1(5.0)$ & $23.9(3.1)$ & $27.2(1.9)$ & $22.4(5.1)$ & $21.6(4.4)$ & $<0.001$ \\
\hline $\begin{array}{l}\text { High WMH or } \geq 2 \\
\text { infarcts }\end{array}$ & 34.0 & 25.0 & 83.3 & 39.3 & 20.0 & 12.9 & 40.0 & $<0.001$ \\
\hline
\end{tabular}

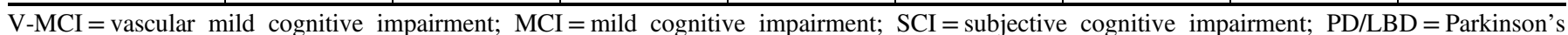
disease/Lewy body dementias.

Values are displayed as percent or mean (standard deviation).

microbleeds, the pattern is described as "mixed". ${ }^{18}$ WMH are visually rated using the Fazekas Scale. ${ }^{15}$ Each scan is visually reviewed for quality. Standard operating procedures have been developed and are stored in a study binder, following FDA guidance for imaging endpoints and guidelines for multi-center brain imaging studies. ${ }^{19}$

Neuroimaging evidence of a potential vascular cause or contribution to cognitive decline is defined, a priori, as either the presence of two or more infarcts or beginning confluent or confluent WMH according to the ARWMC scale on any MRI or CT slice. The ARWMC scale was chosen because it is simple for sites to apply and can be used with either CT or MRI, facilitating site identification of participants for the V-MCI group. Additionally, the MRI-based Fazekas scale will be recorded. The imaging criteria for V-MCI are based on Vas-Cog criteria for pure vascular cognitive impairment, ${ }^{3}$ but to increase sensitivity the criteria were revised to require only two infarcts instead of three and to include beginning confluent WMH instead of confluent $\mathrm{WMH}$, and to increase specificity supratentorial infarcts are required. Post-hoc analyses will test the validity of these criteria for identifying patients at risk of continued cognitive decline. Strategic locations of infarcts were not part of the imaging criteria because the required locations and sizes are not precisely defined in the literature.

A visual assessment of medial temporal atrophy is made using the Scheltens scale, ${ }^{20}$ which is rated from 0 to 4 on each side and was averaged for this analysis. Quantitative segmentation of the hippocampus using high-resolution T1-weighted is planned.

Quantitative analyses of brain images will be batched and performed near the end of the study recruitment period according to the best available methods at that time. The Canadian Dementia Imaging Protocol acquires images suitable for analysis of global and regional brain volumes including cortical thickness; DTI for analysis of white matter connectivity, and multiple BOLD volumes suitable for analyzing resting state functional connectivity.

The data from COMPASS-ND used in this paper, including MRI Digital Communication in Medicine (DICOM) is stored and curated on the Longitudinal Online Research Information System (LORIS). ${ }^{21,22}$

\section{Statistical Analyses, Sample Size, and Data Access Policies}

The study is designed to be an open access platform for secondary analyses of the risk factors, predictors, and cognitive outcomes of common neurodegenerative diseases. Therefore, there is no pre-defined primary outcome.

The V-MCI group sample size of 200 was based on having $80 \%$ power to detect factors that would double the risk of progression to dementia at two years (from $20 \%$ to $40 \%$ ), assuming $20 \%$ loss to follow-up at two years, alpha of 0.05 , and a two-tailed $\mathrm{p}$ value.

Access to COMPASS-ND data will be provided to the worldwide research community according to the policies of the Canadian Consortium on Neurodegeneration in Aging (CCNA; https://ccna-ccnv.ca/policies/). Project proposals should be submitted to the publications and Data Access Committee (PDAC). After study recruitment is completed, data will be provided to the Global Alzheimer's Association Interactive Network (GAAIN) for access through their portal.

To explore the feasibility of recruitment and study measurements, we present descriptive statistics of the distribution of 
Table 4: MRI findings in first 200 participants

\begin{tabular}{|c|c|c|c|c|c|c|c|c|}
\hline Characteristic & All $(n=197)$ & $\begin{array}{l}\text { AD dementia } \\
\quad(n=24)\end{array}$ & $\begin{array}{c}\text { Mixed/V-MCI } \\
\quad(\mathbf{n}=\mathbf{1 8})\end{array}$ & $\operatorname{MCI}(n=84)$ & SCI $(\mathbf{n}=35)$ & PD/LBD $(n=31)$ & Other $(n=5)$ & $P$ value \\
\hline Infarcts-any & 24.9 & 45.8 & 38.9 & 26.2 & 5.7 & 19.4 & 0 & 0.005 \\
\hline Lacunes-any & 14.7 & 12.5 & 33.3 & 19.1 & 2.9 & 6.5 & 0 & 0.02 \\
\hline Non-lacunar-any & 13.7 & 37.5 & 16.7 & 11.9 & 2.9 & 12.9 & 0 & 0.006 \\
\hline CMBs-any & 24.6 & 16.7 & 66.7 & 22.9 & 22.9 & 10.0 & 40.0 & $<0.001$ \\
\hline \multicolumn{9}{|l|}{$\begin{array}{l}\text { Microbleed } \\
\text { pattern }\end{array}$} \\
\hline Probable CAA & 9.6 & 8.3 & 27.8 & 10.8 & 2.9 & 3.3 & 20.0 & 0.02 \\
\hline Possible CAA & 9.6 & 0 & 27.8 & 8.4 & 20.0 & 0 & 0 & \\
\hline Non-lobar & 4.6 & 4.2 & 11.1 & 2.4 & 2.9 & 6.7 & 20.0 & \\
\hline Mixed & 1.5 & 4.2 & 0 & 2.4 & 0 & 0 & 0 & \\
\hline None & 74.4 & 83.3 & 33.3 & 75.9 & 74.3 & 90.0 & 60.0 & 0.01 \\
\hline \multicolumn{9}{|l|}{ ARWMC } \\
\hline None & 5.1 & 12.5 & 0 & 4.8 & 8.6 & 0 & 0 & 0.002 \\
\hline Focal & 64.0 & 62.5 & 27.8 & 59.5 & 74.3 & 87.1 & 60 & \\
\hline $\begin{array}{l}\text { Beginning } \\
\text { confluence }\end{array}$ & 20.3 & 20.8 & 38.9 & 23.8 & 14.3 & 3.2 & 40 & \\
\hline Confluent & 10.7 & 4.2 & 33.3 & 11.9 & 2.9 & 9.7 & 0 & \\
\hline $\begin{array}{l}\text { Medial temporal } \\
\text { atrophy }\end{array}$ & $1.14(0.97)$ & $2.00(0.93)$ & $1.67(0.80)$ & $1.03(0.88)$ & $0.59(0.70)$ & $1.02(0.99)$ & $1.50(1.41)$ & $<0.001$ \\
\hline
\end{tabular}

AD = Alzheimer's disease; V-MCI = vascular mild cognitive impairment; $\mathrm{MCI}$ = mild cognitive impairment; SCI = subjective cognitive impairment; $\mathrm{PD} /$ $\mathrm{LBD}=$ Parkinson's disease/Lewy body dementias; ARWMC = Age-Related White Matter Changes scale; $\mathrm{CMB}=$ cerebral microbleed; WMH $=$ white matter hyperintensity.

Values are percentages or mean (standard deviation).

vascular risk factors and vascular MRI findings in the first 200 recruited participants according to clinical diagnosis. V-MCI and mixed dementia are grouped for analysis. To compare prevalence across groups the chi-square test is used, and to compare medial temporal atrophy scores analysis of variance (ANOVA) is used. The kappa statistic is used to test the reliability of the two readers on 50 COMPASS-ND cases ( 7 with infarcts and 10 with microbleeds), with values $\geq 0.60$ indicating substantial agreement. Agreement was based on the presence or absence of any lesions of that type; there were too few participants with multiple lesions to test the reliability of lesion counts. Because these are interim, exploratory analyses without the full planned sample size, multivariable adjustments are not done and there is no adjustment for multiple comparisons. Analyses were done using SAS version 9.4 (Cary, NC). A p value of $<0.05$ is considered significant.

\section{Results}

The first wave of 200 consenting participants were recruited from 13 sites across Canada. MRI was performed in 197 participants. One participant could not complete the MRI because their head was too large for the coil, one had an MRI incompatible prosthesis that was not recognized at the time of consent, and one declined. MRI were judged adequate for determining infarcts and WMH in all participants. Two participants (1\%) did not have measurement of microbleeds or cSS because of motion (one participant) or susceptibility artifact (one participant).
Table 5: Inter-rater Reliability of COMPASS-ND Measurements

\begin{tabular}{l|c|c}
\hline Measurement & Kappa & 95\% CI \\
\hline Infarcts & 0.68 & $0.42-0.93$ \\
\hline Microbleeds & 0.94 & $0.81-0.99$ \\
\hline Fazekas total score & 0.73 & $0.60-0.87$ \\
\hline MRI criteria for VCI & 0.79 & $0.62-0.96$ \\
\hline
\end{tabular}

Agreement between the two study readers for presence or absence of that lesion type, based on 50 cases.

Characteristics of the 197 participants who completed MRI are shown in Table 3. As expected, distribution of vascular risk factors differed across groups and was highest in the participants with V-MCI or mixed dementia $(n=18)$. The pre-specified radiological criteria for potential vascular contributions to cognitive impairment was seen in 34\% overall, including in 8/10 with $\mathrm{V}-\mathrm{MCI}$ and $7 / 8$ with mixed dementia. Additionally, many participants in the other disease groups (ranging from $12.9 \%$ to $40.0 \%$ ) also met our radiological criteria for a vascular component (Table 3).

Infarcts were seen in $24.9 \%$ (Table 4), including many with dementia due to $\mathrm{AD}$ (11/24). Among the 49 with infarcts the median number was 1 , the maximum was 5 , and the interquartile range was $1-2$. However, most patients with AD had only one infarct. 
Non-lacunar infarcts were not uncommon (13.7\%). Infarcts were least common in participants with subjective cognitive impairment (SCI).

Microbleeds were seen in $25.6 \%$ including $20.0 \%$ with lobar microbleeds and $6.2 \%$ with deep microbleeds (Table 3). Among the 48 with microbleeds, the median was 2 , the maximum was 14 , and the interquartile range was 1-3. Only two participants had cortical superficial siderosis; one with $\mathrm{V}-\mathrm{MCI} /$ mixed dementia and one with MCI.

Beginning confluent or confluent WMH was common (31.0\%; Table 4). Mean Scheltens medial temporal atrophy scores were highest in patients with AD dementia (Table 4).

Reliability of the two readers is shown in Table 5. Reliability was good to excellent for the different lesion types.

Twelve participants $(6.1 \%)$ had incidental non-vascular findings: three suspected meningiomas, two pituitary masses, one presumed lipoma, one intraparenchymal brain tumor, one parotid tumor, one aneurysm, one developmental venous anomaly, one presumed dermoid cyst, and one traumatic encephalomalacia.

\section{Discussion}

The COMPASS-ND study will provide a platform for investigating vascular contributions to neurodegeneration across multiple neurological diseases and syndromes in a prospective cohort study with standardized methods. The preliminary findings demonstrate the feasibility of the study protocols, and that radiological evidence of vascular brain injury was common even in participants recruited without clinical knowledge of vascular comorbidity. These findings concord with neuropathological studies at autopsy showing that mixed pathology with a vascular component is the most common cause of dementia. ${ }^{23}$ Future analyses in the entire dataset will explore the relationship between vascular brain injury and its impact on neurodegeneration across multiple conditions, including when other neurodegenerative diseases are present. This will include analyses of the impact of vascular brain injury on cognition, behavior, activities of living, atrophy, and brain connectivity. Per COMPASS-ND data access policies, the entire de-identified dataset $(n=2310)$ including digital imaging compliant in medicine (DICOM) MRI images will be made available for access by the worldwide research community after study recruitment is completed.

The high prevalence of vascular brain injury in cognitively impaired persons is concordant with findings from autopsy studies in the general population. Even among persons with clinically diagnosed and neuropathologically confirmed $\mathrm{AD}$, vascular pathology is common and is not a mere bystander. A large neuropathological study of the risk for $\mathrm{AD}$ dementia found that macroscopic infarcts accounted for $8.9 \%$, cerebral amyloid angiopathy for $8.1 \%$, atherosclerosis for $6.0 \%$, and arteriolosclerosis for $5.2 \%$ of the risk. ${ }^{24}$ Most prior studies of multiple etiology dementia have focused on the combination of $\mathrm{AD}$ pathology and cerebrovascular disease. The COMPASS-ND study will make an important contribution by enabling the study of vascular contributions to the expression of PD, ${ }^{25} \mathrm{LBD}$, and fronto-temporal lobar degeneration, which so far have attracted less attention.

Some (3/18) of the participants recruited with into the vascular/mixed category did not meet radiological criteria for a vascular contribution (defined as multiple supratentorial infarcts or beginning confluent WMH) according to the Vascular Core Lab review. Potential reasons for this could include a lower local threshold for the degree of vascular brain injury deemed clinically sufficient to impair cognition, the presence of strategic infarcts (which were not included in the Core Lab definition based on lack of research consensus), the presence of clinical non-radiological information known only to the local team (e.g., a history of stroke that was not detected on neuroimaging), or lack of reliability between the local radiology read and the Vascular Core Lab read. Higher local sensitivity for detecting vascular brain injury could also account for this, although we suspect that instead the Vascular Core Lab was more sensitive because the MRI data were acquired using a high quality research protocol and the assessments were done by experienced readers specifically tasked with identifying vascular brain injury using standardized criteria.

We commonly found that participants had radiological evidence of vascular brain injury that may have been sufficient to support a vascular contribution to their cognitive syndrome, including $25 \%$ of the participants recruited into the AD group and $39 \%$ of the participants in the MCI group. These participants could have been recruited into the vascular/mixed group had the vascular component been recognized at the time of clinical diagnosis. Similarly, another cohort study found that $6 \%$ of participants referred to a research study with $\mathrm{AD}$-dementia or AD-MCI had to be reassigned to the VCI group based on infarcts or severe WMH that were not recognized based on their clinical neuroimaging. ${ }^{26}$ This suggests that vascular contributions to neurodegeneration are probably under-recognized in clinical practice.

Clinicians should be careful to assess whether a vascular contribution to neurodegeneration is present in any patient with cognitive symptoms or decline. Given the commonality of mixed disease, this is important even when another contributor such as $\mathrm{AD}$ or PD is already recognized. Our findings support recommendations for neuroimaging in cases of mild cognitive impairment or dementia. ${ }^{2,27}$ However, the treatment of covert cerebrovascular disease is controversial with limited evidence from clinical trials. ${ }^{28}$ At minimum, a history of vascular conditions and risk factors should be taken, blood pressure should be measured, and the pulse checked (to screen for atrial fibrillation). ${ }^{28}$ The use of aspirin could be considered for patients with covert brain infarcts. ${ }^{29}$ Even though definitive clinical trial evidence has not yet been amassed, it nonetheless is likely that vascular disease is the most modifiable contributor to dementia. Anything that prevents stroke will also prevent dementia. ${ }^{30}$

Strengths of this study include the use of a standardized research MRI protocol, a centralized Vascular Core Lab following good clinical practices and FDA guidance for imaging endpoints, and MRI assessments done by qualified, experienced radiologists according to STRIVE criteria. There are also some limitations to the methods. To share the workload the Vascular Core Lab was distributed between two sites; however, we show similar inter-rater reliability of measurements as those achieved by single sites. Quantitative analyses, for example of $\mathrm{WMH}$ volume, have not been completed yet but the data will be made available later to the research community at large. Sequences for measuring brain volume ${ }^{13}$ resting state functional MRI, ${ }^{31}$ and diffusion tensor imaging have also been collected and can be used in later analyses. However, given time and resource constraints some advanced sequences relevant for vascular disease, for example for vascular reactivity and blood brain barrier permeability, ${ }^{32}$ could not be included in the protocol. 
The COMPASS-ND dataset will be an excellent resource for analyzing the vascular contributions to neurodegeneration, both within and across common neurodegenerative illnesses. Analyses are being undertaken currently by COMPASS-ND investigators, but the dataset is open to all investigators subject to COMPASSND data access and publication policies. This first look at our data shows that it is reliable and consistent with neuropathological evidence that vascular brain injury commonly accompanies agerelated neurodegenerative diseases. These findings support the use of neuroimaging in clinical practice to identify potentially preventable covert cerebrovascular disease.

\section{ACKNOWLEDGMENTS}

The study was funded by the Canadian Institutes of Health Research through the Canadian Consortium on Neurodegeneration in Aging.

\section{DisClosures}

Dr. Smith has received consulting fees from Biogen and Alnylam, and royalties from UpToDate. Dr. Duchesne is a shareholder and officer of True Positive Medical Devices Inc. Dr. Black has received consulting fees from Biogen and Roche, and receives research funding for clinical trials from Biogen, Genentech, Roche, Eli Lilly, Novartis, and Optina. The other authors report no conflicts.

\section{Statement of Authorship}

EES analyzed study data, performed statistical analysis, and drafted the manuscript. SD designed the MRI acquisition protocol, analyzed study data, and revised the manuscript critically for intellectual content. FG and FS made study measurements and revised the manuscript critically for intellectual content. VW coordinated the study and reviewed and revised the manuscript critically for intellectual content. CRM maintained study databases, reviewed study data for quality, revised the manuscript critically for intellectual content. RF, SG, RC and MB contributed to study protocols and revised the manuscript critically for intellectual content. SB co-developed the MRI acquisition protocol, analyzed study data and revised the manuscript critically for intellectual content.

\section{SUPPLEMENTARY MATERIAL}

To view supplementary material for this article, please visit https://doi.org/10.1017/cjn.2021.19.

\section{REFERENCES}

1. Chertkow H, Borrie M, Whitehead V, et al. The comprehensive assessment of neurodegeneration and dementia: Canadian cohort study. Can J Neurol Sci. 2019;46(5):1-13.

2. Gorelick PB, Scuteri A, Black SE, et al. Vascular contributions to cognitive impairment and dementia: a statement for healthcare professionals from the American Heart Association/American Stroke Association. Stroke. 2011;42(9):2672-713.

3. Sachdev P, Kalaria R, O'Brien J, et al. Diagnostic criteria for vascular cognitive disorders: a VASCOG Statement. Alzheimer Dis Assoc Disord. 2014;28(3):206-18.

4. Albert MS, DeKosky ST, Dickson D, et al. The diagnosis of mild cognitive impairment due to Alzheimer's disease: recommendations from the National Institute on Aging-Alzheimer's
Association workgroups on diagnostic guidelines for Alzheimer's disease. Alzheimer Dement: J Alzheimer Assoc. 2011;7(3):27079.

5. Wahlund LO, Barkhof F, Fazekas F, et al. A new rating scale for age-related white matter changes applicable to MRI and CT. Stroke. 2001;32(6):1318-22.

6. McKhann GM, Knopman DS, Chertkow H, et al. The diagnosis of dementia due to Alzheimer's disease: recommendations from the National Institute on Aging-Alzheimer's Association workgroups on diagnostic guidelines for Alzheimer's disease. Alzheimer Dement: J Alzheimer Assoc. 2011;7(3):263-69.

7. Rabinovici GD, Carrillo MC, Forman M, et al. Multiple comorbid neuropathologies in the setting of Alzheimer's disease neuropathology and implications for drug development. Alzheimer Dement: Transl Res Clin Interv. 2017;3(1):83-91.

8. Nasreddine ZS, Phillips NA, Bedirian V, et al. The Montreal Cognitive Assessment, MoCA: a brief screening tool for mild cognitive impairment. J Am Geriatr Soc. 2005;53(4):695-99.

9. Morris JC. The Clinical Dementia Rating (CDR): current version and scoring rules. Neurology. 1993;43(11):2412-14.

10. Ismail Z, Aguera-Ortiz L, Brodaty $\mathrm{H}$, et al. The Mild Behavioral Impairment Checklist (MBI-C): a rating scale for neuropsychiatric symptoms in pre-dementia populations. J Alzheimer Dis. 2017;56(3):929-38.

11. Hachinski VC, Iliff LD, Zilhka E, et al. Cerebral blood flow in dementia. Arch Neurol. 1975;32(9):632-37.

12. Duchesne S, Chouinard I, Potvin O, et al. The Canadian dementia imaging protocol: harmonizing national cohorts. J Magn Reson Imaging. 2019;49(2):456-65.

13. Potvin O, Chouinard I, Dieumegarde L, et al. The Canadian dementia imaging protocol: harmonization validity for morphometry measurements. NeuroImage Clin. 2019;24:101943.

14. Wardlaw JM, Smith EE, Biessels GJ, et al. Neuroimaging standards for research into small vessel disease and its contribution to ageing and neurodegeneration. Lancet Neurol. 2013;12(8): 822-38.

15. Fazekas F, Chawluk JB, Alavi A, Hurtig HI, Zimmerman RA. MR signal abnormalities at $1.5 \mathrm{~T}$ in Alzheimer's dementia and normal aging. Am J Roentgenol. 1987;149(2):351-56.

16. Cordonnier C, Potter GM, Jackson CA, et al. Improving interrater agreement about brain microbleeds: development of the Brain Observer MicroBleed Scale (BOMBS). Stroke. 2009;40(1):94-99.

17. Linn J, Halpin A, Demaerel P, et al. Prevalence of superficial siderosis in patients with cerebral amyloid angiopathy. Neurology. 2010;74(17):1346-50.

18. Pasi M, Charidimou A, Boulouis G, et al. Mixed-location cerebral hemorrhage/microbleeds: underlying microangiopathy and recurrence risk. Neurology. 2018;90(2):e119-26.

19. Wiseman SJ, Meijboom R, Valdes Hernandez MDC, et al. Longitudinal multi-centre brain imaging studies: guidelines and practical tips for accurate and reproducible imaging endpoints and data sharing. Trials. 2019;20(1):21.

20. Korf ES, Wahlund LO, Visser PJ, Scheltens P. Medial temporal lobe atrophy on MRI predicts dementia in patients with mild cognitive impairment. Neurology. 2004;63(1):94-100.

21. Mohaddes Z, Das S, Abou-Haidar R, et al. National neuroinformatics framework for Canadian Consortium on Neurodegeneration in Aging (CCNA). Front Neuroinform. 2018;12:85.

22. Das S, Zijdenbos AP, Harlap J, Vins D, Evans AC. LORIS: a webbased data management system for multi-center studies. Front Neuroinform. 2011;5:37.

23. Schneider JA, Arvanitakis Z, Bang W, Bennett DA. Mixed brain pathologies account for most dementia cases in communitydwelling older persons. Neurology. 2007;69(24):2197-204.

24. Boyle PA, Yu L, Leurgans SE, et al. Attributable risk of Alzheimer's dementia attributed to age-related neuropathologies. Ann Neurol. 2019;85(1):114-24.

25. Ramirez J, Dilliott AA, Binns MA, et al. Parkinson's disease, NOTCH3 genetic variants, and white matter hyperintensities. Mov Disord. 2020;35(11):2090-95.

26. Kapoor A, Bartha R, Black SE, et al. Structural brain magnetic resonance imaging to rule out comorbid pathology in the assessment of Alzheimer's disease dementia: findings from the Ontario 
Neurodegenerative Disease Research Initiative (ONDRI) study and clinical trials over the past 10 years. J Alzheimer Dis. 2020;74(3):747-57.

27. Ismail Z, Black SE, Camicioli R, et al. Recommendations of the 5th Canadian Consensus Conference on the diagnosis and treatment of dementia. Alzheimer Dement: J Alzheimer Assoc. 2020;16(8):1182-95.

28. Smith EE, Saposnik G, Biessels GJ, et al. Prevention of stroke in patients with silent cerebrovascular disease: a scientific statement for healthcare professionals from the American Heart Association/American Stroke Association. Stroke. 2017;48(2):e44-71.

29. Smith EE, Barber P, Field TS, et al. Canadian Consensus Conference on Diagnosis and Treatment of Dementia
(CCCDTD)5: guidelines for management of vascular cognitive impairment. Alzheimer Dement: Transl Res Clin Interv. 2020; 6(1):e12056

30. Hachinski V, Einhaupl K, Ganten D, et al. Preventing dementia by preventing stroke: the Berlin Manifesto. Alzheimer Dement: J Alzheimer Assoc. 2019;15(7):961-84.

31. Badhwar A, Collin-Verreault Y, Orban P, et al. Multivariate consistency of resting-state fMRI connectivity maps acquired on a single individual over 2.5 years, 13 sites and 3 vendors. Neuroimage. 2020;205:116210.

32. Smith EE, Beaudin AE. New insights into cerebral small vessel disease and vascular cognitive impairment from MRI. Curr Opin Neurol. 2018;31(1):36-43. 